\title{
On the Validity of the Imbert-Fick Law: Mathematical Modelling of Eye Pressure Measurement
}

\author{
Gabriela González Castro' ${ }^{1}$ Alastair D. Fitt ${ }^{2}$, John Sweeney ${ }^{{ }^{*}}$ \\ ${ }^{1}$ IRC in Polymer Science and Technology, University of Bradford, Bradford, UK \\ ${ }^{2}$ Oxford Brookes University, Oxford, UK \\ Email: "j.sweeney@bradford.ac.uk
}

Received 23 November 2015; accepted 20 March 2016; published 23 March 2016

Copyright (C) 2016 by authors and Scientific Research Publishing Inc.

This work is licensed under the Creative Commons Attribution International License (CC BY). http://creativecommons.org/licenses/by/4.0/

c) (7) Open Access

\begin{abstract}
Ophthalmologists rely on a device known as the Goldmann applanation tonometer to make intraocular pressure (IOP) measurements. It measures the force required to press a flat disc against the cornea to produce a flattened circular region of known area. The IOP is deduced from this force using the Imbert-Fick principle. However, there is scant analytical justification for this analysis. We present a mathematical model of tonometry to investigate the relationship between the pressure derived by tonometry and the IOP. An elementary equilibrium analysis suggests that there is no physical basis for traditional tonometric analysis. Tonometry is modelled using a hollow spherical shell of solid material enclosing an elastic liquid core, with the shell in tension and the core under pressure. The shell is pressed against a rigid flat plane. The solution is found using finite element analysis. The shell material is anisotropic. Values for its elastic constants are obtained from literature except where data are unavailable, when reasonable limits are explored. The results show that the force measured by the Goldmann tonometer depends on the elastic constant values. The relationship between the IOP and the tonometer readings is complex, showing potentially high levels of inaccuracy that depend on IOP.
\end{abstract}

\section{Keywords}

Tonometry, Finite Element Analysis, Imbert-Fick Law

\section{Introduction}

Intraocular pressure (IOP)—-the difference between atmospheric pressure and the pressure inside the eye-is a

${ }^{*}$ Corresponding author.

How to cite this paper: Castro, G.G., Fitt, A.D. and Sweeney, J. (2016) On the Validity of the Imbert-Fick Law: Mathematical Modelling of Eye Pressure Measurement. World Journal of Mechanics, 6, 35-51.

http://dx.doi.org/10.4236/wjm.2016.63005 
parameter of key importance in assessing the health of the eye. For the human eye IOP values in the range 1.6 $3.3 \mathrm{kPa}(12$ - $25 \mathrm{mmHg}$ ) are considered normal. Higher values are always a cause of concern since they may indicate glaucoma, a serious optic nerve neuropathy that may lead to blindness.

A device called a tonometer is used to measure IOP. A large variety of tonometer designs are in use, which give fast and non-invasive measurements at varying levels of accuracy. The instrument considered to be the "gold standard" by the ophthalmic community, and the focus of this study, is the Goldmann Applanation Tonometer ("GAT"). When a GAT is used, a flat metal disc of a known, standardized diameter is brought into contact with the cornea and pressed against it until the whole area of the disc is in contact and "applanation" (observed via a slit lamp) is judged to have taken place. The force on the disc required to achieve full contact is measured, so that the mean pressure on the disc surface can be calculated. Routinely, the tonometer measurement is interpreted by equating this measured pressure with the IOP. The assumption embodied in this interpretation is known as the Imbert-Fick law, which asserts that every gm force required for applanation corresponds to 1 $\mathrm{mmHg}$ IOP (from which it may easily be calculated that the diameter of the applanation disc is $3.06 \mathrm{~mm}$ ). This paper is an evaluation of the Imbert-Fick law, for which there appears to be no theoretical justification. We shall first demonstrate a simple equilibrium analysis that shows that the law is at best an estimation technique that may correlate with IOP. We then proceed to use a numerical technique to give a critical review of the relationship between tonometry measurements and IOP.

From a mathematical perspective, the tonometry problem may be modelled using solid mechanics. We shall adopt the finite element approach in which the model is three-dimensional. We perform calculations on a sphere with a linear elastic solid outer shell containing a material that is essentially an elastic fluid. Pressure is applied to the inner material by prestraining the outer shell. Then, boundary conditions are applied by introducing a plane surface touching the sphere's outer surface, corresponding to the tonometer, and applying equilibrating displacements to at the opposite surface of the sphere to compress it against the plane.

The work is divided as follows: Section 1.1 outlines a brief introduction to the anatomical, physiological, clinical and pathological facts relevant to the study of the IOP. Section 2 presents the analysis, including details of the finite element models used and the material elastic properties. The results are presented and discussed in Section 3, where the tonometer performance is evaluated, and finally conclusions follow in Section 4.

\section{Anatomical, Physiological, and Clinical Considerations}

According to Fatt and Weissman [1], the human eye is approximately spherical, with a typical diameter of 25 $\mathrm{mm}$. Its outer wall comprises the cornea and the sclera (which are joined at the limbus). The interior of the eye is mostly constituted by the vitreous body, except for the region comprising the anterior chamber and the lens. The sclera is internally coated by the membrane known as the retina. Figure 1 presents a schematic representation of the human eye showing the main components.

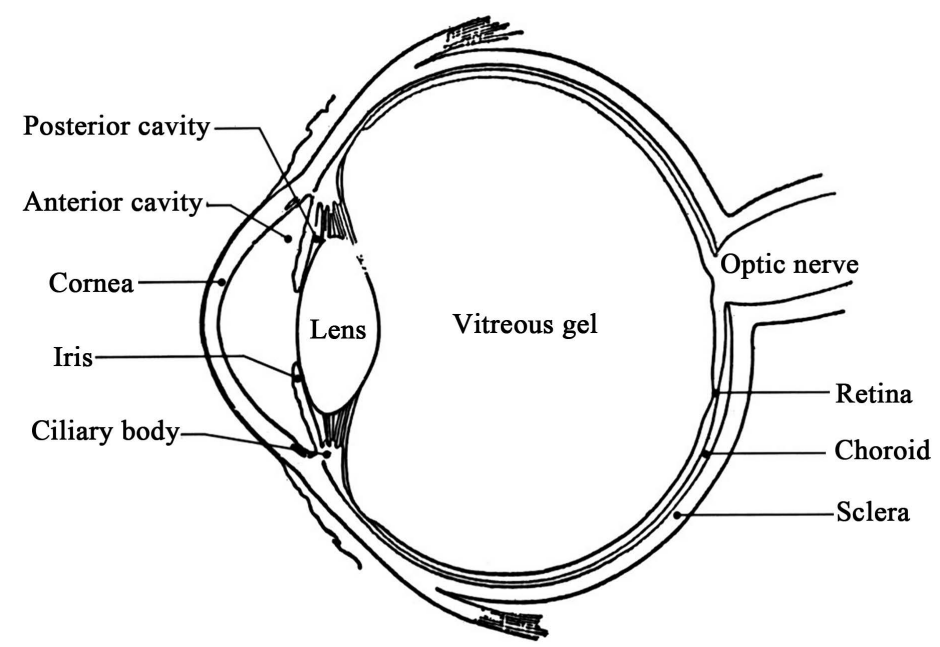

Figure 1. Schematic representation of the human eye showing its main components. (http://www.wpclipart.com/medical/anatomy/eye). 
The cornea is a transparent organ composed mostly of water and collagen. It possesses a high content of nerve fibres but almost entirely lacks blood vessels. This organ makes up almost one sixth of the outer surface area of the eye. The principal function of the cornea is optical since it is thought to be responsible for as much as $70.0 \%$ of the total refractive power of the eye due to its transparency; however its mechanical properties also make it capable of resisting the intraocular pressure and providing a protective coat for the eye. The cornea's protective qualities are attributed to the collagen fibres within this organ [2], which are in turn associated with its known anisotropic nature [3]. The thickness of the cornea varies across it, the thinnest region being located at the centre with a typical value of $0.52 \mathrm{~mm}$. Corneal thickness increases progressively towards the limbus where it reaches a typical value of $0.70 \mathrm{~mm}$.

The sclera is a tissue commonly known as "the white" of the eye and comprises the rest of the outer shell of the eye. This tissue is composed of approximately a third of interwoven elastic collagen fibres, which are responsible for its elasticity, and two thirds of water. The main function of the sclera is to protect the intraocular organs from injury and damage; it is stiff enough to resist the IOP. The thickness of the sclera varies with age as well as location. For an adult healthy human eye, Fatt and Weissman [1] report that the thickness of the sclera varies from $1.0 \mathrm{~mm}$ around the optic nerve to $0.33 \mathrm{~mm}$ at the rectus muscle insertions.

The vitreous body is a transparent gel-like substance comprising $80 \%$ of the total volume of the eye. Among the functions attributed to the vitreous body are its ability to both work as an optical medium, and to retain the retina. (The retina is attached to the choroid, which in turn is attached to the sclera.)

Though a scientific foundation for the Imbert-Fick law is largely lacking, the Goldmann-type applanation tonometer has nevertheless been designed under the assumption that this law is valid. The Imbert-Fick law seems to have been used for the first time for tonometer design in the last decade of the nineteenth century by Imbert [4] and Fick [5], but it was not until the 1950s that Goldmann designed the GAT with its applanation tip of diameter $3.06 \mathrm{~mm}$ as described above. It has now become standard to use a tip of these dimensions as this simplifies its use for the physician and avoids numerical conversions that could lead more readily to human errors.

When reflecting on the applanation process, it soon becomes clear that the Imbert-Fick law cannot perfectly describe it. During the applanation process, the cornea is flattened, and this in itself must require a force to create sufficient bending stress, even in the absence of any IOP. The effect of the surface tension of the tear liquid on the cornea surface must also be accounted for. This latter effect has been calculated to be equivalent to an added $0.06 \mathrm{kPa}$ tension on the tonometer [6], a small matter in comparison with the normal IOP range of 1.6 $3.3 \mathrm{kPa}$. It has been claimed that, for normal IOP values with a standard Goldmann tonometer, the bending and surface tension effects cancel out [6]-[9]. In view of the small effect of surface tension, we shall henceforth exclude it in the analysis below.

\section{Analysis}

\subsection{Equilibrium Considerations}

To fix ideas, in Figure 2 we analyse a pressurised sphere compressed by opposing forces $F$ along a diameter. A fluid under pressure $p$ ( $p=$ IOP) is contained by a thin shell under a tension $\sigma_{t}$ in all tangential directions. To gain insight into the relationship between pressure, shell stress and compressing force, we create a free body consisting of the hemisphere in Figure 2. At the upper flat surface only normal stresses act so that, using the areas to convert stresses to forces while assuming a thin $(t \ll R)$ sphere, equilibrium of vertical forces gives

$$
F+2 \pi R t \sigma_{t}-\pi R^{2} p=0
$$

For $F=0$ this gives the standard solution for a pressurised thin sphere [10]. Suppose the force $F$ is distributed over a circular area of radius $a$. Then the upward force can be re-expressed as a compressive contact stress $\sigma_{c}$ such that $\sigma_{c}=F / \pi a^{2}$. In terms of this stress, Equation (1) becomes

$$
\sigma_{c}+\frac{2 R t}{a^{2}} \sigma_{t}-\left(\frac{R}{a}\right)^{2} p=0 .
$$

In contrast, the Imbert-Fick law is 


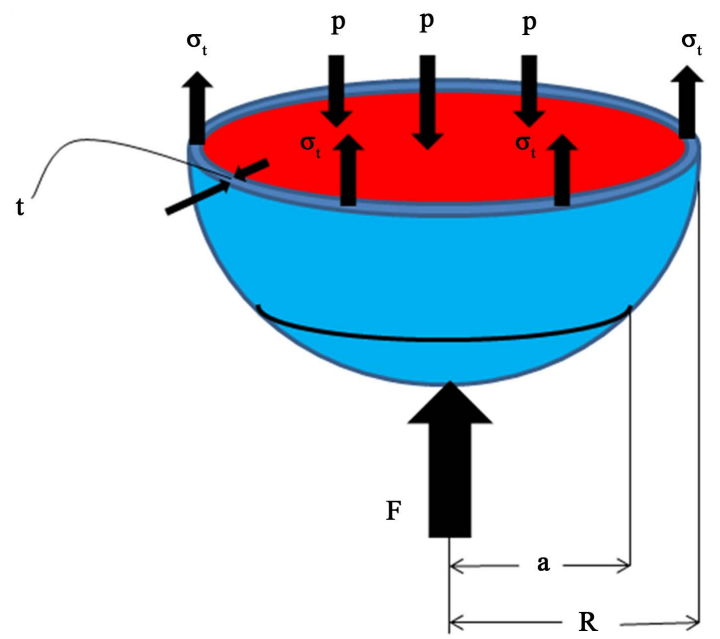

Figure 2. Free body diagram of compressed and pressurised sphere.

$$
\sigma_{c}-p=0
$$

Further insight can be gained by creating a cylindrical section with radius $r<R$, again with the tonometer contact area of radius a centred on the vertical symmetry axis, as shown in Figure 3. The cylindrical cut exposes a shear stress $\tau$, that is generally nonzero, being the resolved component of the tensile shell stress. Equilibrium of vertical forces gives

$$
F+2 \pi r T \tau-\pi r^{2} p=0 .
$$

$F$ is assumed to be distributed about the contact area radius $a$, to give the mean contact pressure $\sigma_{c}$ :

$$
\sigma_{c}+\frac{2 r T \tau}{a^{2}}-\left(\frac{r}{a}\right)^{2} p=0 .
$$

This equation demonstrates the extreme case where $p$ is small enough to be neglected. Then, $\sigma_{c}>p, \tau<0$. This value of $\sigma_{c}$ corresponds to that required to flatten the cornea. This corresponds to the observation of Orssengo and Pye [11], that the applanating and intraocular pressures will in general be unequal, as a significant level of force is required to flatten the cornea, irrespective of any pressure behind it.

With no applanation $\sigma_{c}=0$ and $\tau>0$. On applanation, as $\sigma_{c}$ increases, $\tau$ decreases. Considering the case $r=a$, as long as $\tau$ remains positive, $\sigma_{c}<p$. However, when the stress required to bend the cornea is the dominant factor as discussed above, $\sigma_{c}>p$ and $\tau<0$. These findings are confirmed by the numerical studies reported below.

The above reasoning suggests that Equation (3) is not generally valid. Śródka [12] has shown that incorporation of surface tension effects is not effective at correcting this model, and raises some additional objections, including the elastic stability of the applanating sphere.

Equations (2) and (5) indicate that $\sigma_{c}$ will be correlated positively with $p$, especially given the customary use of a standard value of area in applanation tonometry. The equations cannot be used to evaluate $p$ directly, as $\sigma_{t}$ and $\tau$ are unknown. Solutions to the problem obtained by numerical means are described below.

\subsection{Finite Element Modelling}

\subsubsection{Background}

An early instance of the use of the finite element method to model the mechanical behaviour of the eye is due to Buzard [13]. He created an axisymmetric model in the form of a pressurised shell which was used to simulate the radial expansion of the eye in response to intraocular pressure. The simulations were compared with experiments in which the radial expansion of human cadaver eyes that were pressurised up to $12 \mathrm{kPa}$ was measured. More recently, Asejczyk-Widlicka et al. [14] produced an axisymmetric shell model of the eye and compared predicted dimensional changes with experiment. While the utility and credibility of the finite element method in this context was established, in neither case was modelling of tonometry attempted. 


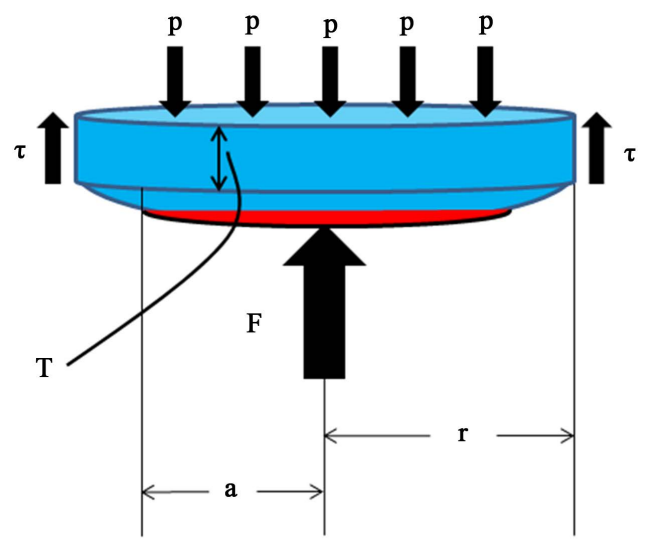

Figure 3. Free body diagram of cylindrical section of cornea and pressure fluid.

Elsheikh et al. [15] and Elsheikh and Wang [6] have modelled the tonometry process using the finite element method. Their model involved the cornea only, which was represented by part of a sphere of appropriate radius. The models had a circular boundary that was subject to boundary conditions designed to simulate connection to the sclera. The corneal material was assumed to be nonlinearly elastic (specifically hyperelastic) and initially isotropic. Different workers have made fundamentally varying assumptions about the mechanical behaviour of the cornea; Singh et al. [16] assumed it to be anisotropic and linear elastic, whereas Pandolfi and Manganiello [17] assumed it to be both nonlinear and anisotropic. There is experimental evidence for nonlinear mechanical behaviour of both cornea and sclera [14] [17], though it has also been reported that nonlinear effects are not observed in the eye below IOP values of $4 \mathrm{kPa}$ [6] [18]. Measurements show that the sclera is highly anisotropic [19] and this is consistent with the fibrous composite material model studied by Girard et al. [18]. The simplest feasible modelling assumption seems to be that of anisotropic linear elasticity, provided that the IOP values are kept below a $4 \mathrm{kPa}(=30 \mathrm{mmHg})$ upper limit: this is how we will proceed below.

\subsubsection{Mesh Geometry and Boundary Conditions}

We have created in-house software that generates input files that, when operated upon by the finite element package ABAQUS [20], produce models of multilayered spheres. Typical finite element meshes are shown in Figures 4-6. The shell and interior are assigned different material properties with respectively solid elastic and fluid-like characteristics. Large deformation analyses were implemented.

As in Figure 2, compression is along the vertical axis. Each model is run in two stages:

- First, the sphere is pressurised. This is done by exploiting the thermal expansion capability of ABAQUS. A thermal expansion coefficient is assigned to the shell material and a fictitious temperature drop imposed. The temperature drop/expansion coefficient combination is adjusted to give an appropriate pressure level in the interior. We investigated three pressure levels: 1.2, 2.1 and $3.3 \mathrm{kPa}(6,15.75$ and $24.75 \mathrm{mmHg})$.

- Secondly, displacement boundary conditions are imposed on a circular region of nodes on the top nodes centred around the vertical axis. They are all displaced downwards by the same value. The lower surface of the sphere rests on a flat rigid analytical surface that is held stationary. As the displacement increases, the circular contact area increases and the value of the contact area is output.

The accuracy of the contact area calculation depends on the areas of the elements on the lower surface. Elements are concentrated in the contact zone for this reason. Two basic methods for this were used. In the first (type A) shown in Figure 5, the tangential side lengths of successive pairs of elements are in a constant ratio (>1, in this case 4/3) on moving away from the central vertical axis. In the other method (type B), shown in Figure 6, the mesh density in the central third of the lower quadrant is increased but kept to a constant element size within the denser region. Method A has the characteristic that, as the analysis progresses, the elements newly making contact at the outer region of the contact field are of increasing area. This causes some increase in the error in the area calculation. However, the two refinement methods produced results that were not significantly different from each other.

All the models possess fourfold rotational symmetry about the vertical axis, containing at the centre a cube of elements from which vectors are produced and on which nodes are positioned. The number of elements in the 


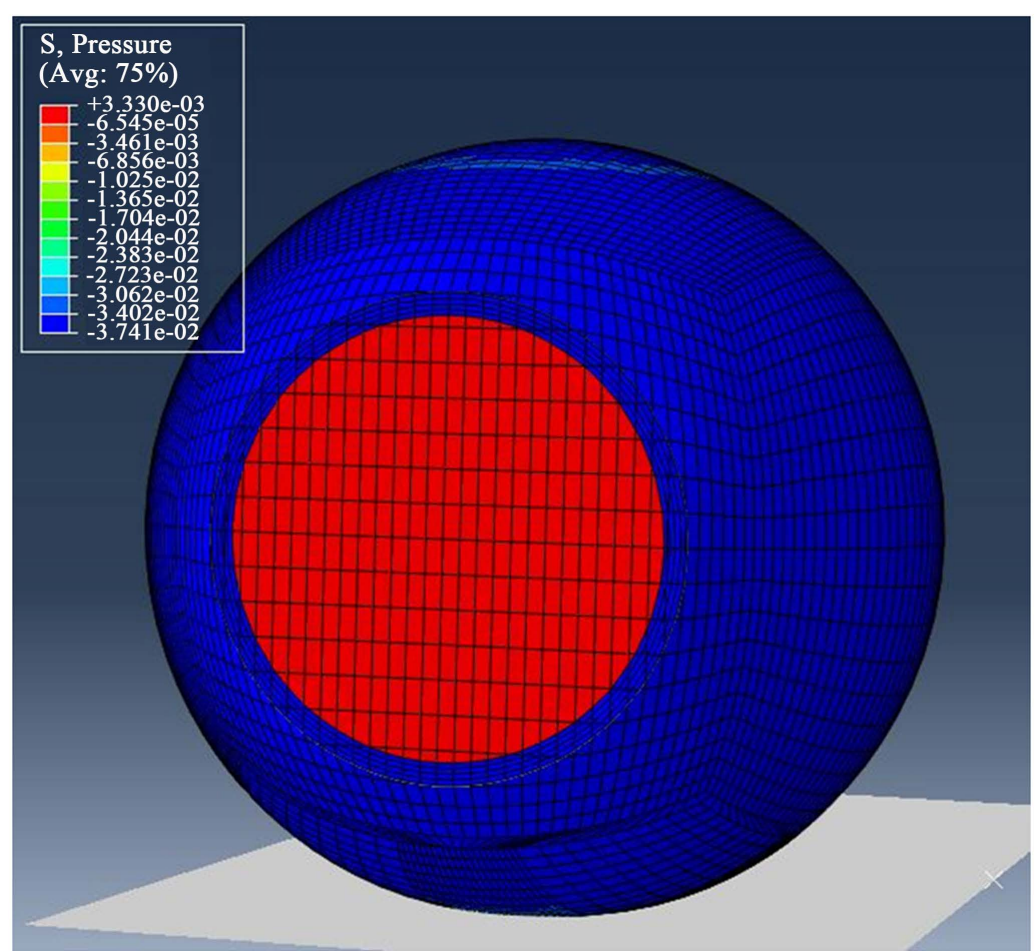

Figure 4. General view of a multilayered sphere model compressed onto the lower flat surface, sectioned to show pressurised interior.

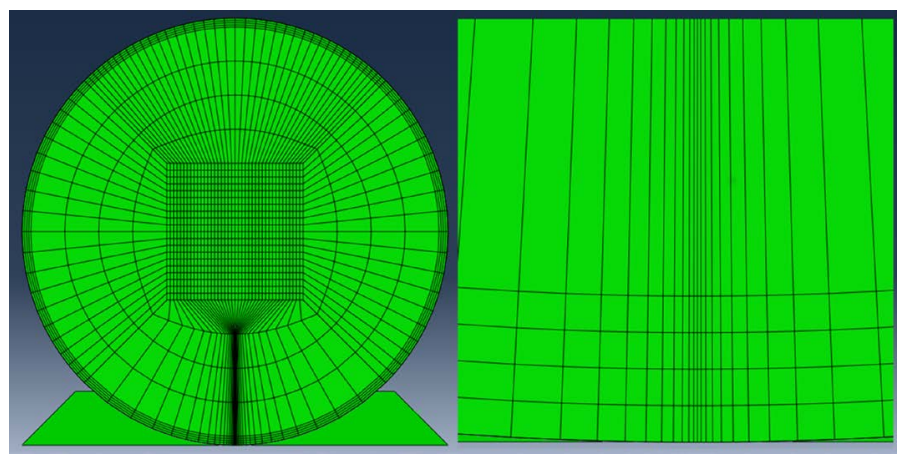

Figure 5. Left: cross section of sphere model, showing a central cube of $20 \times 40 \times 40$ elements and the concentration of elements at the contact surface (type A refinement). Right: close-up of contact surface. The four outer rows of elements are shell material.

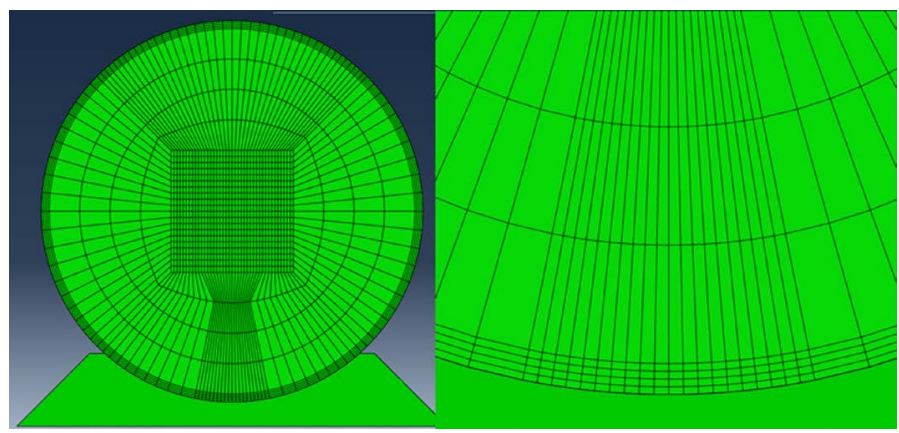

Figure 6. Left: cross section of sphere model, showing a central cube of $20 \times 40 \times 40$ elements and element density increased by a factor of 4 in the contact region (type B refinement). Right: close-up of contact area. 
cube controls the mesh density. In the meshes shown, the cube is an array of $20 \times 40 \times 40$ elements, with a $40 \times$ 40 face opposite the contact area. The model contains a total of 70,400 solid elements. Meshes with cube arrays $20 \times 30 \times 30$ (45,200 elements) were also generated for comparison and to establish mesh convergence. Eight-noded linear hexahedral elements (C3D8) were used throughout. To correspond to typical human eye dimensions, the outer radius of the model was $12.5 \mathrm{~mm}$ and the inner radius of the shell was $11.98 \mathrm{~mm}$. The displacement boundary condition was applied to nodes on the top surface within a circular area of radius $5 \mathrm{~mm}$ centred on the vertical symmetry axis.

\subsubsection{Material Properties}

For the (liquid) core material a compressible Gaussian model was used, as defined by the strain energy density function $W$ :

$$
W=\mu\left(\left(\lambda_{I}^{*}\right)^{2}+\left(\lambda_{I I}^{*}\right)^{2}+\left(\lambda_{I I I}^{*}\right)^{2}-3\right)+B(J-1)^{2}
$$

$J$ is the volume ratio defined by

$$
J=\lambda_{I} \lambda_{I I} \lambda_{I I I}
$$

where $\lambda_{I}, \lambda_{I I}$ and $\lambda_{I I I}$ are the principal extension ratios and the starred quantities, which are the deviatoric extension ratios, are given by

$$
\lambda_{i}^{*}=\lambda_{i} J^{-1 / 3}(i=I, I I, I I I) .
$$

In Equation (6) $\mu$ and $B$ are material parameters, with $\mu$ governing shape change and $B$ controlling volume change. With $\mu \ll B$ we have a material that resists volume change but has very little resistance to shape change, approximating closely to an elastic liquid. We therefore use the values

$$
\mu=0.1 \mathrm{kPa}, B=200 \mathrm{MPa}
$$

which ensure that the stresses within the core material are essentially hydrostatic.

We treat the outer shell-the scleral and corneal regions-as linear elastic solids. In view of the observed nonlinear behaviour [6] [18], we shall restrict the IOP values to a maximum of $3.3 \mathrm{kPa}$ (=24.75 $\mathrm{mmHg}$ ), below the level of $4 \mathrm{kPa}(=30 \mathrm{mmHg})$ at which the nonlinearity becomes significant.

Outside the region of the cornea, the outer layer of the eye consists mainly of sclera of the order of $1 \mathrm{~mm}$ thick, with an added thickness of $\sim 0.08 \mathrm{~mm}$ of choroid. Modulus values of the sclera and choroid are given respectively as 2.3 and 0.6 MPa (Friberg and Lace [21], quoted by Keeling et al. [22]). A rule-of-mixtures calculation then gives a value of 2.2 MPa for the sclera/choroid composite; clearly, the sclera is the dominant factor. Schulz et al. [23] presented tensile results from human sclera and arrived at a modulus value $2.6 \pm 2 \mathrm{MPa}$. Girard et al. [18] have deduced modulus values for rhesus monkey sclera using a combined experimental and modelling approach, and found evidence of nonlinear behaviour, with stiffness increasing with IOP. They found the stiffness to be in the range 1.9 - 3.5 MPa for an IOP of $10 \mathrm{~mm} \mathrm{Hg}$, and 3.4 - 4.7 MPa at $30 \mathrm{mmHg}$. On the basis of these various observations we shall use the value 2.4 MPa for this elastic modulus of the human sclera in the all of the modelling described below.

There is evidence that the corneal modulus is smaller than that of the sclera [13]. Singh et al. [16] have summarized the work of four authors who reported stress-strain experiments on excised strips of cornea, giving Young's modulus values in the range 0.08 - $20 \mathrm{MPa}$. We select an intermediate value of $0.3 \mathrm{MPa}$. This is consistent with that of Zeng et al. [24] which was based on an average of ten measurements, and similar to the lower range of the values derived by Hoeltzel et al. [25].

The default assumption that elastic materials are isotropic is an attractive one, and this premise has been used before in the modelling of the eye (e.g. [6] [15]). However, the sclera is a fibre composite that is anisotropic, with fibres aligned preferentially normal to the eye radius [18], giving a low value of stiffness along a radius. Given the experimental methods employed - the stretching of strips excised from the eye-the modulus values summarised so far relate to circumferential material directions normal to a radius of an ideal spherical eye. Measurements of the modulus of sclera along the radial direction were carried out by Battaglioli and Kamm [19], who found radial values of modulus lower than the circumferential values by a factor of up to 100. Anisotropy in the cornea has also been established Jayasuriya et al. [3]. There is a clear need to allow for anisotropic material behaviour for both the cornea and sclera. We choose to model the material using orthotropic linear elasticity. 
In the spherical polar system of axes defined in Figure 7, we may define circumferential moduli $E_{\theta}$ and $E_{\phi}$ and a radial modulus $E_{r}$ together with three independent Poisson ratios $v_{\phi \theta}, v_{r \theta}$ and $v_{r \phi}$. Then Hooke's law may be stated as

$$
\left(\begin{array}{c}
\varepsilon_{\theta} \\
\varepsilon_{\phi} \\
\varepsilon_{r} \\
\varepsilon_{r \phi} \\
\varepsilon_{r \theta} \\
\varepsilon_{\phi \theta}
\end{array}\right)=\left(\begin{array}{cccccc}
1 / E_{\theta} & -v_{\phi \theta} / E_{\theta} & -v_{r \theta} / E_{\theta} & 0 & 0 & 0 \\
-v_{\phi \theta} / E_{\theta} & 1 / E_{\phi} & -v_{r \phi} / E_{\phi} & 0 & 0 & 0 \\
-v_{r \theta} / E_{\theta} & -v_{r \phi} / E_{\phi} & 1 / E_{r} & 0 & 0 & 0 \\
0 & 0 & 0 & 1 / G_{r \phi} & 0 & 0 \\
0 & 0 & 0 & 0 & 1 / G_{r \theta} & 0 \\
0 & 0 & 0 & 0 & 0 & 1 / G_{\phi \theta}
\end{array}\right)\left(\begin{array}{c}
\sigma_{\theta} \\
\sigma_{\phi} \\
\sigma_{r} \\
\sigma_{r \phi} \\
\sigma_{r \theta} \\
\sigma_{\phi \theta}
\end{array}\right)
$$

which may be rewritten as

$$
\varepsilon=S \sigma
$$

where $S$ is termed the compliance matrix. We shall further assume that the shell material is transversely isotropic with the isotropic planes normal to a radius. Then [26] we can apply the simplifications

$$
\begin{aligned}
& E_{\phi}=E_{\theta} \\
& v_{r \phi}=v_{r \theta} \\
& G_{r \phi}=G_{r \theta}
\end{aligned}
$$

and for the isotropic plane the shear modulus is given by

$$
G_{\phi \theta}=\frac{E_{\theta}}{2\left(1+v_{\phi \theta}\right)} .
$$

There are now five independent elastic constants that must be specified. We identify $E_{\theta}$ with the values 2.4 MPa for the sclera and 0.3 MPa for the cornea discussed above. For values of $E_{r}$ we refer to the work of Battaglioli and Kamm [19], in which experiments on human sclera compressed in the radial direction gave values of approximately $E_{r}=0.04 \mathrm{MPa}$ and $v_{r \theta}=0.47$; we adopt these values for both the sclera and the cornea. Though direct measurements for the other parameter do not appear to be available, Li and Tighe [27] have developed models of the cornea based on fibre composite theory. From their results we may deduce that $v_{\phi \theta} \approx 0.1$, from which we may derive the value of $G_{\phi \theta}$ using Equation (11). The final independent parameter is $G_{r \theta}$, which is important as it will affect the bending stiffness of the shell and consequently its response to applanation. There appear to be no data for this parameter. We can reasonably assume that its value will lie between that of

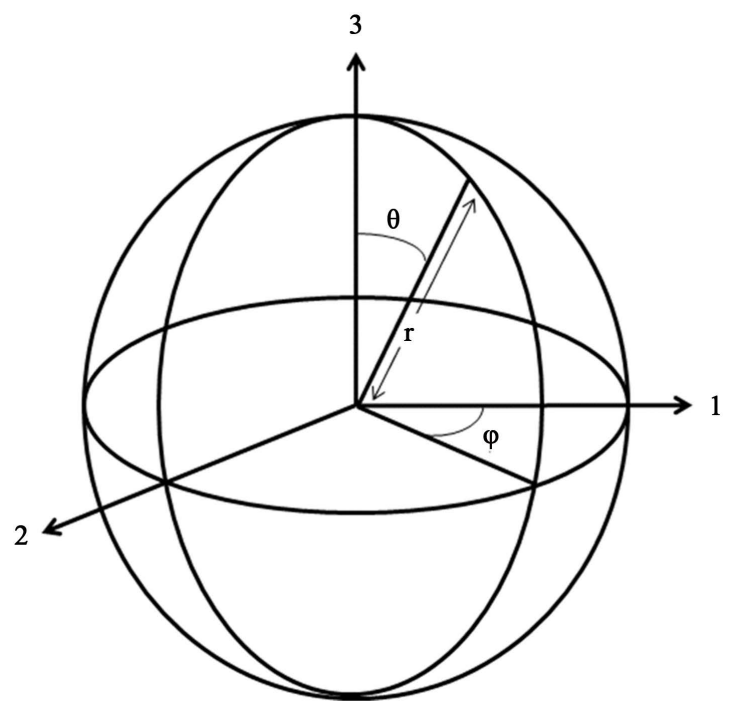

Figure 7. Spherical polar co-ordinate system. 
$G_{\phi \theta}$ and the value of shear modulus for an isotropic plane with modulus $E_{r}$ and Poisson's ratio $v_{r \theta}$. This gives $0.014 \leq G_{r \theta} \leq 1.10$ MPa for the sclera and $0.014 \leq G_{r \theta} \leq 0.27$ for the cornea. In the analyses to follow, a range of values will be used and the implications explored.

To summarise, the values may be expressed as the compliance matrix S of Equation (9) as follows. For the cornea:

$$
S=\left(\begin{array}{cccccc}
3.33 & -0.333 & -1.567 & 0 & 0 & 0 \\
-0.333 & 3.33 & -1.567 & 0 & 0 & 0 \\
-1.567 & -1.567 & 25.0 & 0 & 0 & 0 \\
0 & 0 & 0 & 1 / G_{r \theta} & 0 & 0 \\
0 & 0 & 0 & 0 & 1 / G_{r \theta} & 0 \\
0 & 0 & 0 & 0 & 0 & 3.667
\end{array}\right)(\mathrm{MPa})^{-1}
$$

where $0.014 \leq G_{r \theta} \leq 0.27 \mathrm{MPa}$

For the sclera:

$$
S=\left(\begin{array}{cccccc}
0.417 & -0.0417 & -0.196 & 0 & 0 & 0 \\
-0.0417 & 0.417 & -0.196 & 0 & 0 & 0 \\
-0.196 & -0.196 & 25.0 & 0 & 0 & 0 \\
0 & 0 & 0 & 1 / G_{r \theta} & 0 & 0 \\
0 & 0 & 0 & 0 & 1 / G_{r \theta} & 0 \\
0 & 0 & 0 & 0 & 0 & 0.917
\end{array}\right)(\mathrm{MPa})^{-1}
$$

where $0.014 \leq G_{r \theta} \leq 1.10 \mathrm{MPa}$. For input into ABAQUS the stiffness matrix $D$ given by $D=S^{-1}$ is used.

During applanation, the mechanical properties local to the deformation-those of the cornea-are significant to the process, whereas the scleral properties are of little significance. Therefore, our principal results will be for models for which the whole of the shell has been assigned cornea properties. However, we will present results for models with sclera properties in the whole of the shell also, to illustrate the importance of the mechanical properties to the tonometry results.

\section{Results and Discussion}

Here we compare the pressure on the contacting rigid plane with the internal pressure in the model. During each simulation downward displacements were applied incrementally to the top surface causing the contact area on the lower surface and the associated reaction force to increase. The analyses were continued until the contact area was approximately twice that of the tonometer, and pressure results taken at the tonometer area of 7.354 $(=\pi \times(3.06 / 2)) \mathrm{mm}^{2}$. In the results to be presented below, it is clear that there is consistently some slight nonlinearity, with the slope of the contact area-contact force curve increasing slightly with area. The force at the tonometer area was derived from a quadratic polynomial fit to this curve; this is more accurate than relying on a single point calculation, as there is some degree of scatter in the area evaluation. The IOP is calculated during the analysis, and increases slightly, but not significantly, by only up to less than $1 \%$ when applanated at the tonometer area.

The limits for $G_{r \theta}$ given in the previous section account for a wide range of behaviour. We shall explore the ranges $0.02 \leq G_{r \theta} \leq 0.27 \mathrm{MPa}$ for the cornea material of Equation (12) and $0.02 \leq G_{r \theta} \leq 1.1 \mathrm{MPa}$ for the sclera material of Equation (13). This will provide useful insights into the pressure dependence of the tonometer response, while still leaving some uncertainty in the absolute values it provides. For $G_{r \theta}<0.02 \mathrm{MPa}$, numerical difficulties were encountered and so the lower limit was fixed at this value rather than at $0.014 \mathrm{MPa}$; this is not a significant issue as the trend in behaviour is clear.

\subsection{Cornea Properties}

In Figure 8 and Figure 9 a model with mesh generated via a $20 \times 40 \times 40$ element cube using Type B mesh 


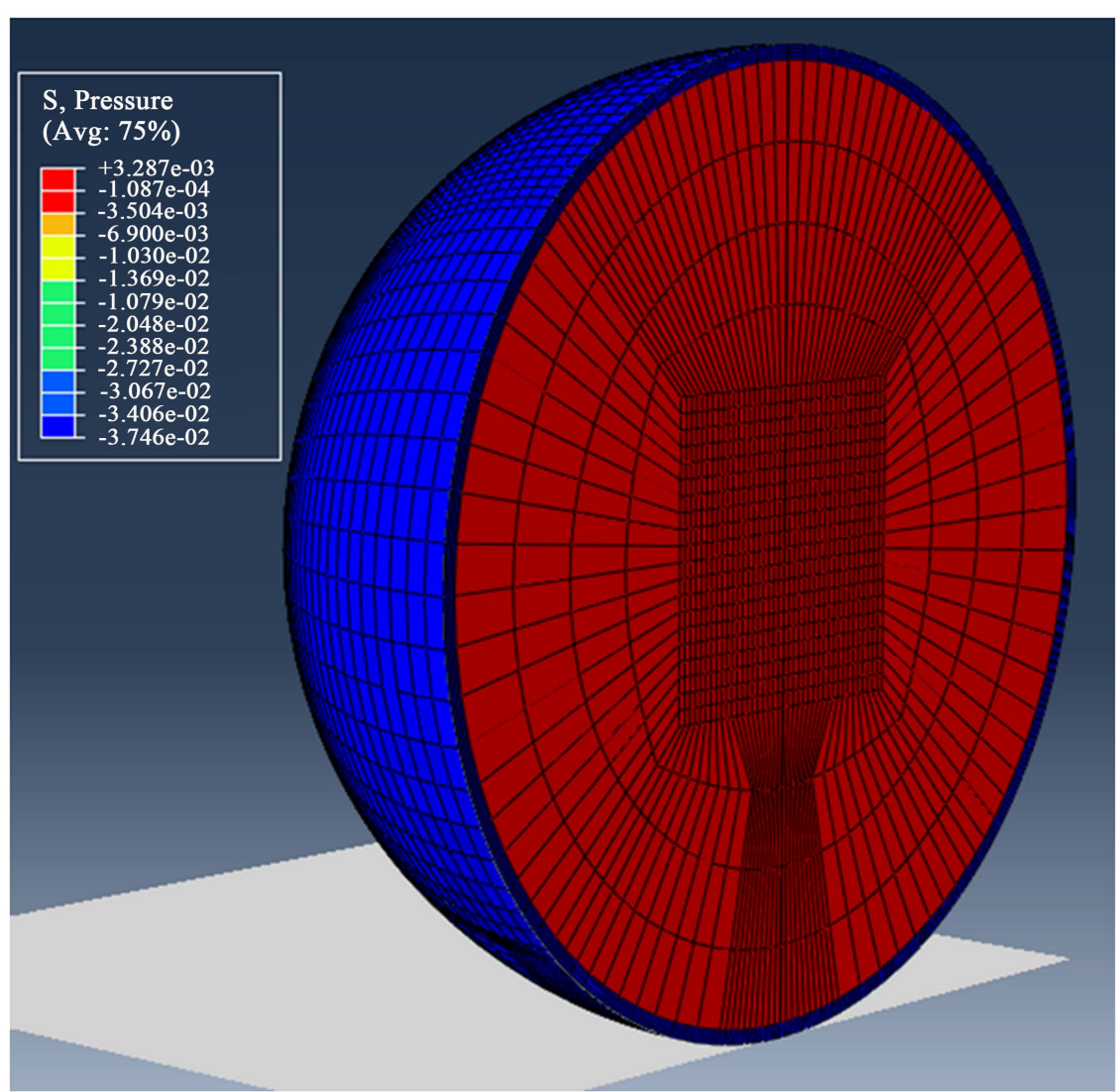

Figure 8. Cross section of pressurised and uncompressed model at $3.3 \mathrm{kPa}$ internal pressure. Mesh type B, based on $20 \times 40 \times 40$ element cube.

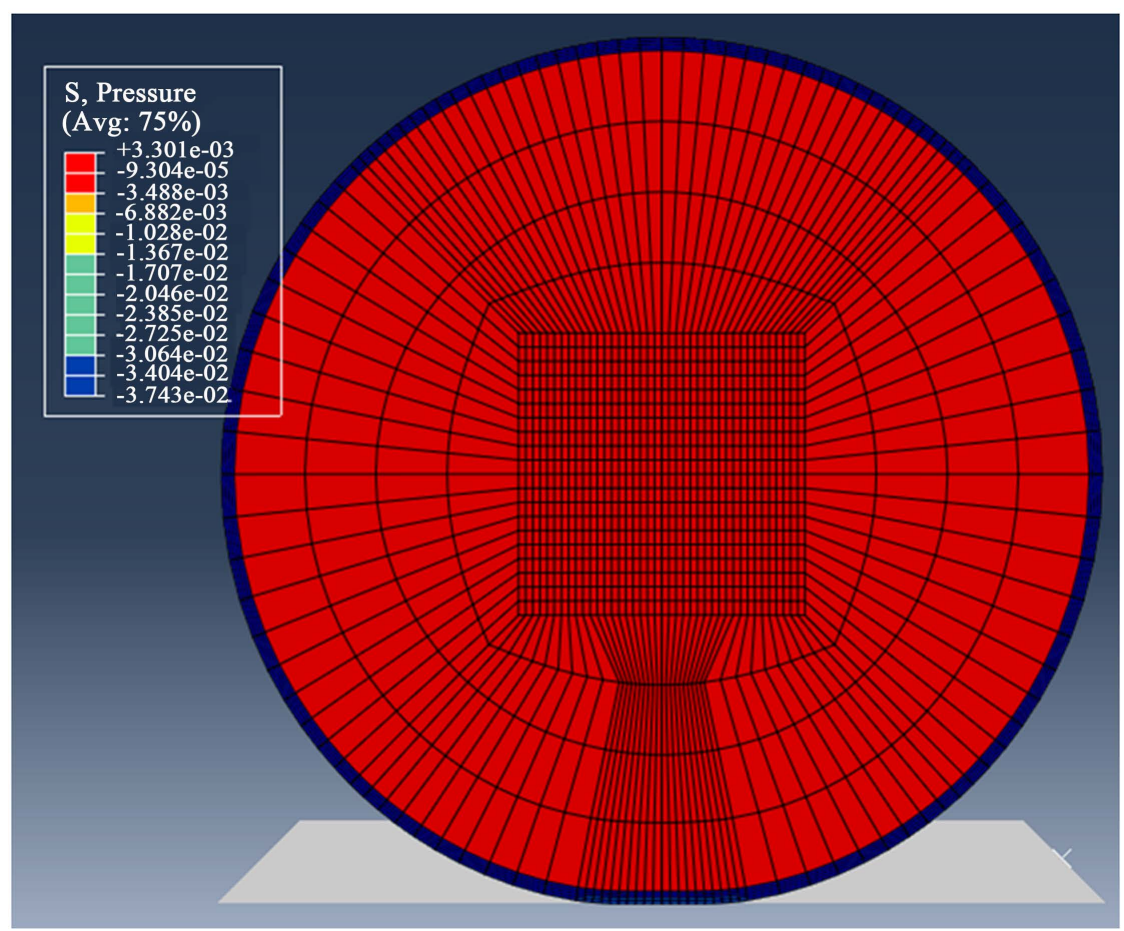

Figure 9. Cross section of the model when compressed to give a contact area of $7.3 \mathrm{~mm}^{2}$. 
refinement is used. This model, in which the internal pressure is $3.3 \mathrm{kPa}$, shows typical behaviour. Other mechanical properties are given in Section 2, with the shell being assigned the cornea properties of Equation (12) and the value of $G_{r \theta}$ assumed to be $0.03 \mathrm{MPa}$. The cross section of Figure 8 shows the state after pressurisation before compression, and Figure 9 shows the compressed state when an applanated area of $7.3 \mathrm{~mm}^{2}$ has been attained.

The response in terms of compression force versus contact area is shown in Figure $\mathbf{1 0}$ for this model and for two other, differently meshed models using the same physical conditions. Points are plotted for each solution increment. The contact area does not increase completely smoothly owing to the discrete size of elements. In Figure 11 we show the response at a lower pressure $1.3 \mathrm{kPa}$ when the value $G_{r \theta}=0.27 \mathrm{MPa}$ is used. Using

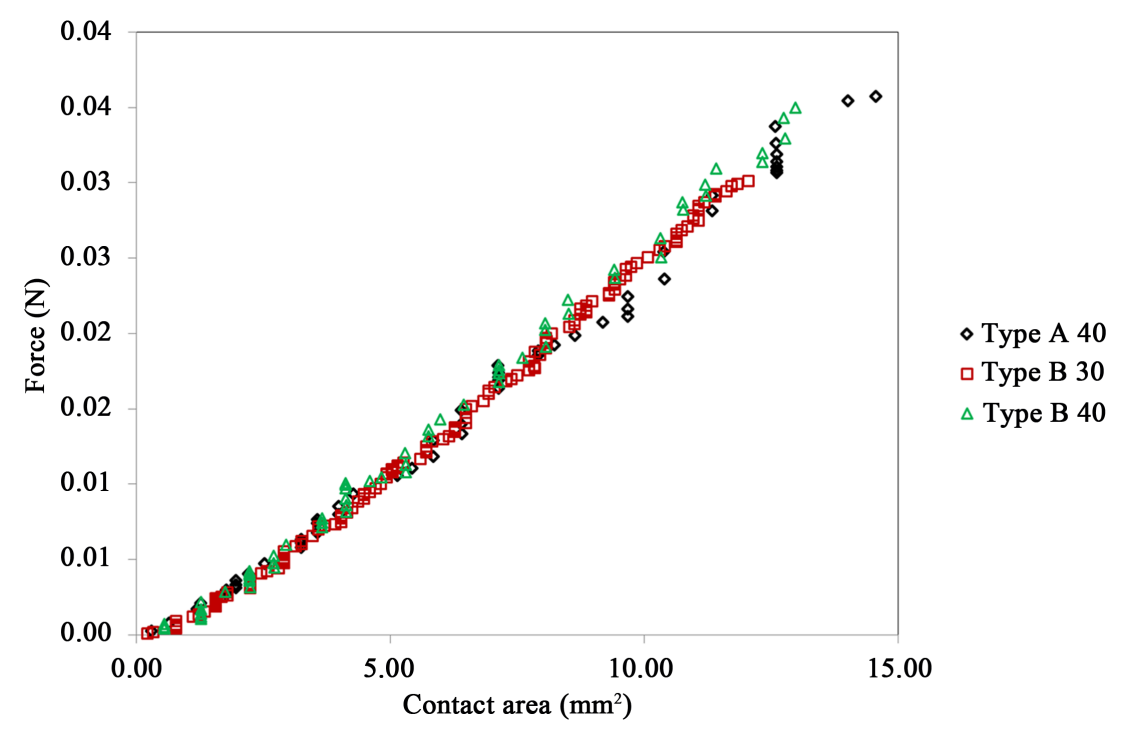

Figure 10. Contact area-force response with IOP $3.3 \mathrm{kPa}$ and, properties of Equation (12) with $G_{r \theta}=0.03 \mathrm{MPa}$. Results are compared for different meshing strategies. Type A 40: Type A mesh refinement, $20 \times 40 \times 40$ inner cube (Figure 5); Type B 30: Type B mesh refinement, $20 \times$ $30 \times 30$ I nner cube; Type B 40; Type B mesh refinement, $20 \times 40 \times 40$ inner cube (Figure 6).

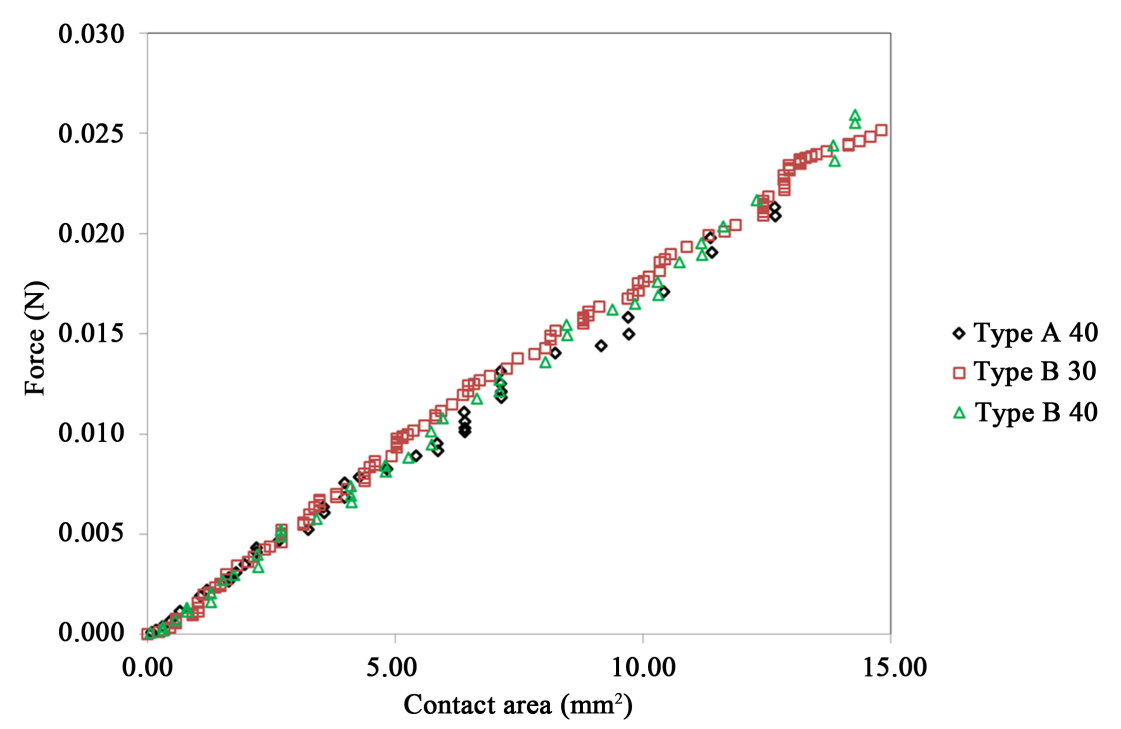

Figure 11. Contact area-force response with IOP $1.3 \mathrm{kPa}$, properties of equation (12) and $G_{r \theta}=0.27 \mathrm{MPa}$. Results are compared for different meshing strategies. Type A 40: Type A mesh refinement, $20 \times 40 \times 40$ inner cube (Figure 5); Type B 30: Type B mesh refinement, $20 \times$ $30 \times 30$ inner cube; Type B 40: Type B mesh refinement, $20 \times 40 \times 40$ inner cube (Figure 6). 
quadratic fitting for each set of data we obtain the force on the tonometer at the contact area $7.354 \mathrm{~mm}^{2}$ and calculate the tonometer pressure. For the case of Figure 10 where the IOP is $3.3 \mathrm{kPa}$, we obtain the figure 2.25 $\mathrm{kPa}$ for Type A $20 \times 40 \times 40$ mesh, $2.27 \mathrm{kPa}$ for the Type B $20 \times 30 \times 30$ based mesh and $2.37 \mathrm{kPa}$ for the Type B $20 \times 40 \times 40$ based mesh. For the case of Figure 11 where the IOP is $1.3 \mathrm{kPa}$, the tonometer pressures for the Type A $20 \times 40 \times 40$, Type B $20 \times 30 \times 30$ and Type B $20 \times 40 \times 40$ are respectively $1.68,1.81$ and $1.72 \mathrm{kPa}$. There is more error at the lower pressure, where the two more finely meshed models differ in their tonometer pressure prediction by $2.4 \%$. The corresponding error for the fine meshes at the higher pressure is $5.2 \%$. While significant in numerical terms the errors here are insufficient to influence our conclusions on the performance of the tonometer; in both cases the tonometer pressures differ markedly from the IOPs. Type A meshes give significantly noisier responses in Figure 10 and Figure 11 than do type B meshes. In Figure 11 the denser Type B $20 \times 40 \times 40$ mesh gives a closer match to the Type A result than does the Type B $20 \times 30 \times 30$, evidence that, as expected, the denser mesh gives a more accurate result. For this reason we shall adopt the Type B $20 \times 40 \times$ 40 mesh as the standard for the models that generate the results presented below.

We also made similar simulations using Type B mesh refinement at IOP values in the range $1.2-3.3 \mathrm{kPa}$ and assuming $G_{r \theta}$ values of 0.02, 0.03, 0.04, 0.1 and 0.27 MPa. The results are summarised in Figure 12.

Tonometer pressure is observed to be an approximately linear function of IOP with a positive intercept. The intercept can be associated with the force required purely to flatten the cornea, increasing with increasing $G_{r \theta}$. The slopes are in all cases less than unity, in the range 0.5 - 0.6, showing another dissimilarity with the ImbertFick law. A fortuitous value of $G_{r e}$ may produce an accurate tonometer pressure, but significant under- or over-prediction seems inevitable over any appreciable IOP range.

A detailed appraisal of the stresses local to the applanation zone helps to understand the physical mechanisms involved, particularly in terms of the free body diagram of Figure 3 and Equations (4) and (5). In the following figures we show stresses in the cornea at the contact zone. The stresses are in $\mathrm{r}-\theta$ co-ordinates, but at small angles to the vertical for purposes of visualisation we can associate stresses $\sigma_{r}$ and $\sigma_{r} \theta$ with vertical forces. A positive value of $\sigma_{r \theta}$ is associated with a downward force.

In Figure 13 we show stresses in the central plane of the sphere near to the contact zone, for the case where $G_{r \theta}=0.02 \mathrm{MPa}$ and IOP $=1.3 \mathrm{kPa}$ for the standard applanation area of $7.35 \mathrm{~mm}^{2}$. Figure 12 shows that this case is accurately predicted by the Imbert-Fick law. In Figure 13, in the left hand image radial stresses $\sigma_{r}$ are not constant on the contact plane, being greater in magnitude nearer the centre. In this region the contact stress is greater than the IOP, at around $1.5-1.6 \mathrm{kPa}$, but at the outer regions of the zone they are less than the IOP. This is consistent with the mean compressive contact stress $\sigma_{c}$ (see Equation (5)) being at around the IOP, $p$. We also show shear stresses $\sigma_{r \theta}$ equivalent to the stress $\tau$ in Equation (5) in the right image of Figure 13. These stresses

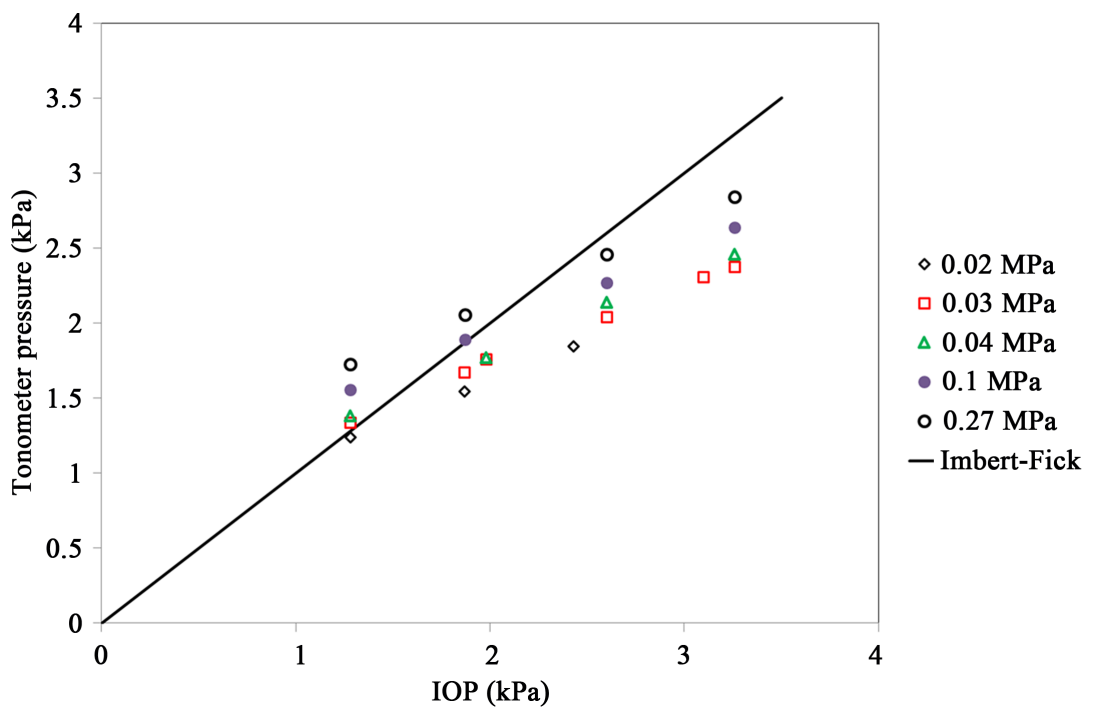

Figure 12. Predictions of tonometer pressure versus IOP for cornea shell properties of Equation (12). The key defines values of $G_{r \theta}$. 

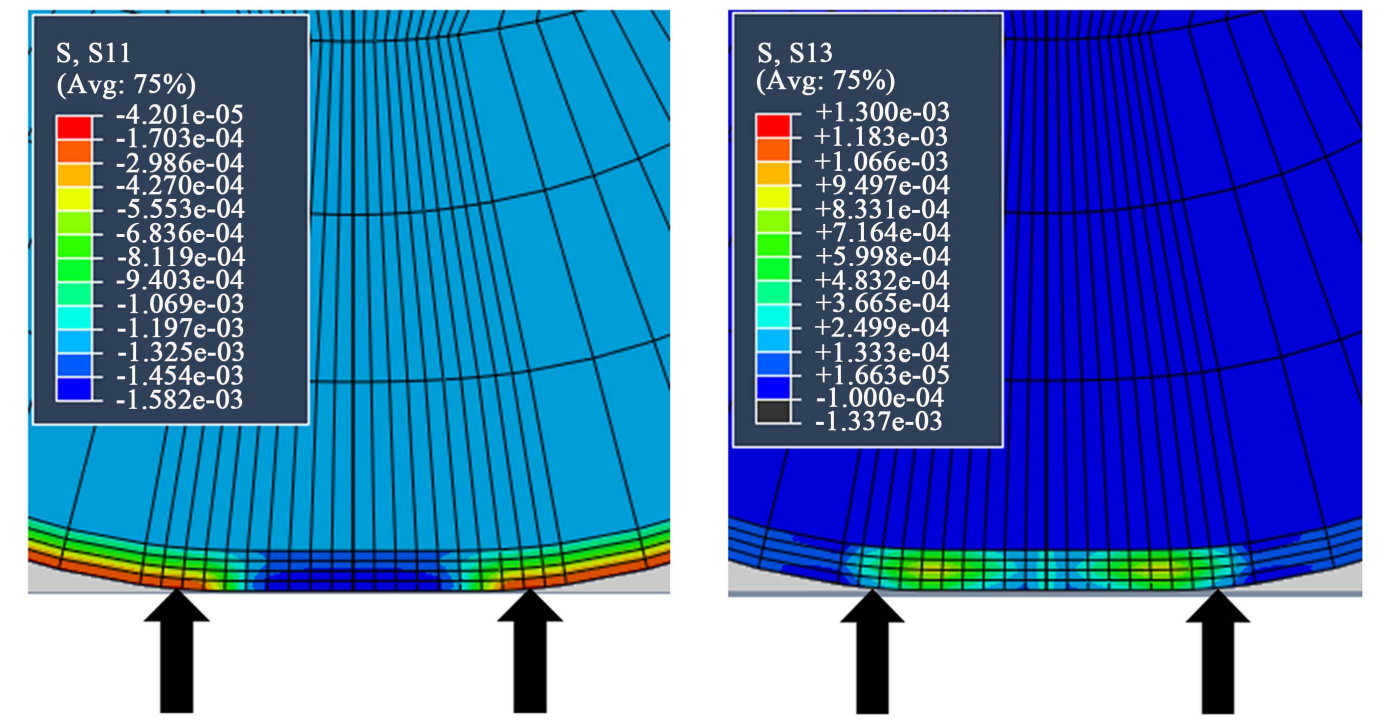

Figure 13. Arrows define the extent of the contact zone. $G_{r \theta}=0.02 \mathrm{MPa}$ and IOP $=1.3 \mathrm{kPa}$. On left, S11 refers to the stress $\sigma_{r}$ in $\mathrm{MPa}$. On right, $\mathrm{S} 13$ refers to the shear stress $\sigma_{r}$ in $\mathrm{MPa}$.

change sign around the edges of the contact zone. This is consistent with IOP $=p-\sigma_{c}$ in Equation (5) when $r=\mathrm{a}$.

Turning attention to cases that are not accurately predicted by Imbert-Fick, consider the case $G_{r \theta}=0.03 \mathrm{MPa}$ and IOP $=3.3 \mathrm{kPa}$. The IOP is under-predicted by our tonometry simulation as shown in Figure 12. The local conditions are shown in Figure 14. The radial stress in the contact region in the left hand image of Figure 14 is approximately equal to the IOP over the central region of the contact zone; however, it is lower in absolute value than the IOP in the outer regions, giving a mean value of contact stress $\sigma_{c}$ lower than IOP, corresponding to the low tonometer pressure shown in Figure 12. In the right image of Figure 14 at the outside edges of the contact area the shear stress is negative, corresponding to a positive value of $\tau$ in Equation (5), and $\sigma_{c}<p$ when $r=a$.

We now consider the case $G_{r \theta}=0.27 \mathrm{MPa}$ and IOP $=1.3 \mathrm{kPa}$, for which the simulated tonometer pressure in Figure 12 is greater than the IOP. The contact stresses are shown on Figure 15. In the left image of Figure 15 the contact stress is greater than the IOP in the central area of the contact zone and lower in outer regions, consistent with an average contact stress $\sigma_{c}$ higher than IOP. The shear stresses in the contact area in the right image of Figure 15 are positive just outside the contact area. We conclude that is consistent with an average shear stress $\tau$ that is downwards (negative), so that Equation (5) corresponds to $\sigma_{c}>p$ and $\tau<0$ when $r=a$.

The above detailed examination of the contact stresses explains how the Imbert-Fick law is capable of both under-predicting and over-predicting the IOP.

\subsection{Scleral Properties}

To evaluate the effect of varying the mechanical properties of the shell, we performed a similar exercise using the scleral properties of Equation (13). The stiffer material gives rise to a broader range of variation of tonometer pressure as a function of $G_{r \theta}$, which we have varied between 0.02 and $1.1 \mathrm{MPa}$. The results are shown in Figure 16.

The slopes of the data now lie in the range $0.4-0.5$, similar to those in Figure 12. The main difference is the greater sensitivity to $G_{r \theta}$, with gross errors apparent at, and in excess of, $G_{r \theta}=0.1 \mathrm{MPa}$. This suggests that for a cornea outside the normal human stiffness range, IOPs predicted by GAT readings could be highly misleading.

\section{Effect of Corneal Radius}

All of the results that have been discussed so far have been obtained with purely spherical models. This is a simplification as in reality (see Figure 1) the cornea has a radius of curvature that is significantly smaller than 

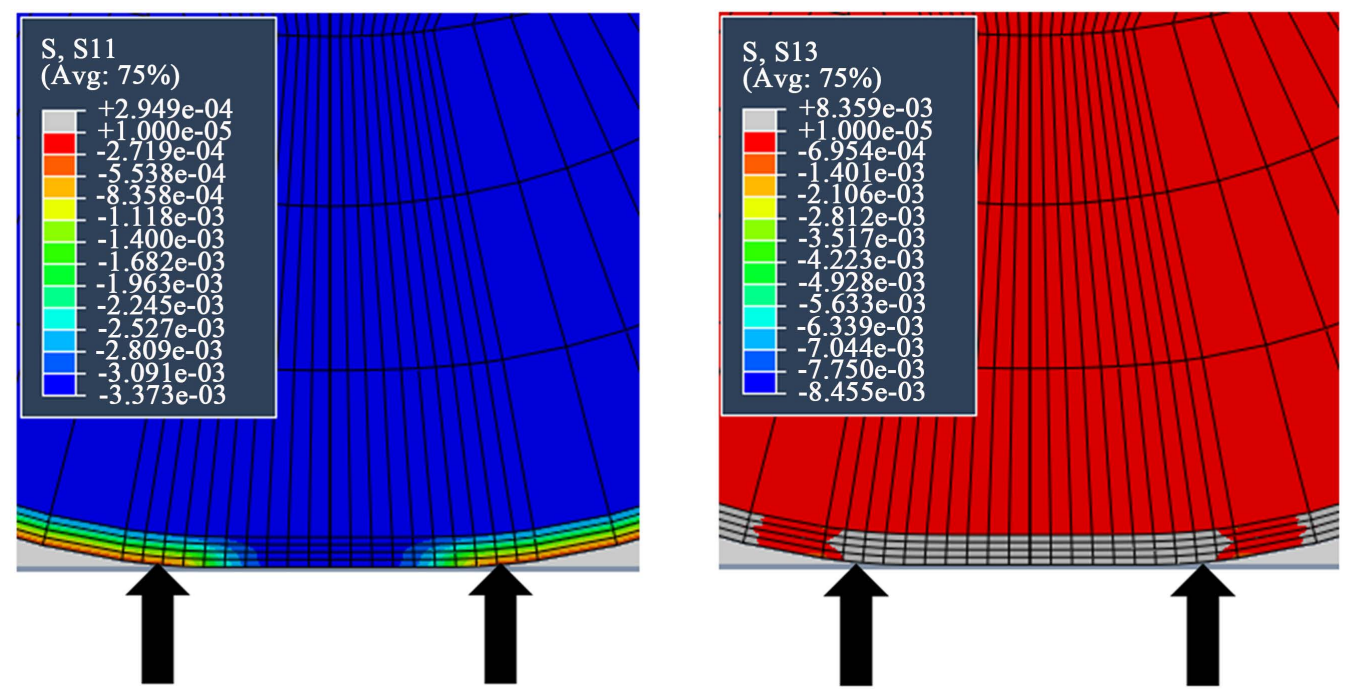

Figure 14. Arrows define the extent of the contact zone. $G_{r \theta}=0.03 \mathrm{MPa}$ and IOP $=3.3 \mathrm{kPa}$. On left S11 refers to the stress $\sigma_{r}$ in $\mathrm{MPa}$. On right $\mathrm{S} 13$ refers to the shear stress $\sigma_{r \theta}$ in $\mathrm{MPa}$.
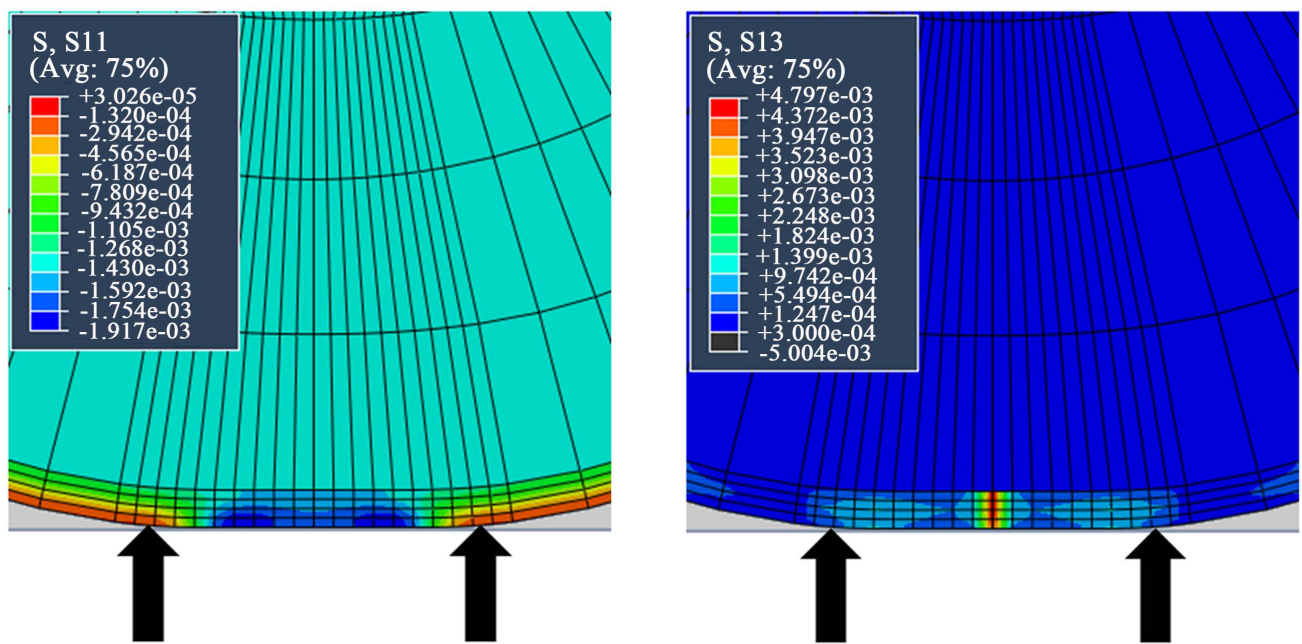

Figure 15. Arrows define the extent of the contact zone. $G_{r \theta}=0.27 \mathrm{MPa}$ and IOP $=1.3 \mathrm{kPa}$. On left S11 refers to the stress $\sigma_{r}$ in MPa. On right $\mathrm{S} 13$ refers to the shear stress $\sigma_{r} \theta$ in MPa.

the radius of the eyeball. To explore the effects of the corneal radius we have created a series of smaller spherical models with radii in a range relevant to the human cornea. The applanation of these spheres resembles locally the applanation of a cornea of realistic size forming part of an eyeball. The shell thickness is kept the same as in the previous models, and the meshing used is type B, based on $20 \times 40 \times 40$ element cube, as shown in Figure 8 and Figure 9.

In Figure 17 we show model tonometer pressures for radii in the range $6.25-12.5 \mathrm{~mm}$, which includes the range $7.2-8.4 \mathrm{~mm}$ which is realistic for the human cornea [15]. The tonometer pressure shows similar functional dependence on the radius for both large and small values of $G_{r \theta}$. For both values of $G_{r \theta}$, we observe a drop in tonometer pressure of approximately $10 \%$ as the radius changes from 7.2 to $8.4 \mathrm{~mm}$. This effect is very similar to that observed in the modelling of Elsheikh et al. [15] at an IOP of $2.0 \mathrm{kPa}(=15 \mathrm{mmHg})$.

While the effect of radius is clearly significant, we can conclude that it will not fundamentally affect the functional dependences of tonometry pressure on IOP as seen in Figure 12 and Figure 13. A lower radius at a value realistic for a cornea will tend to shift the tonometry pressures in Figure 12 upwards by around 20\%; this does not materially affect our conclusions on the accuracy of the tonometry measurements. 


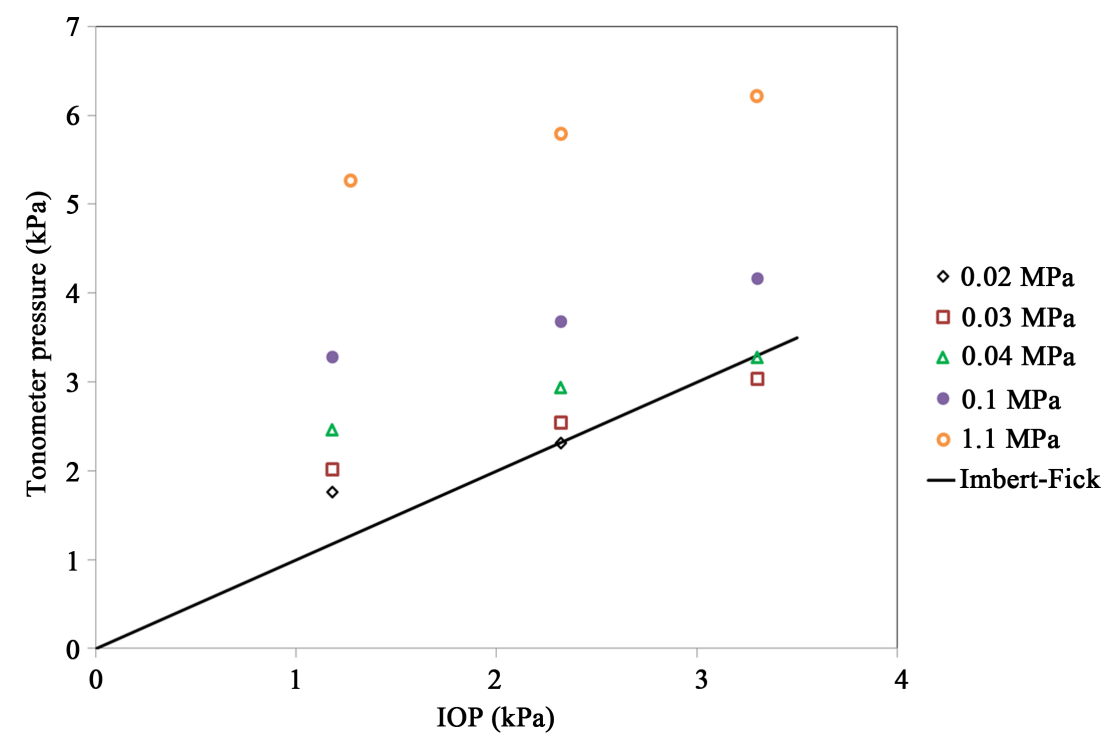

Figure 16. Predictions of tonometer pressure versus IOP for sclera shell properties of Equation (13). The key defines values of $G_{r \theta}$.

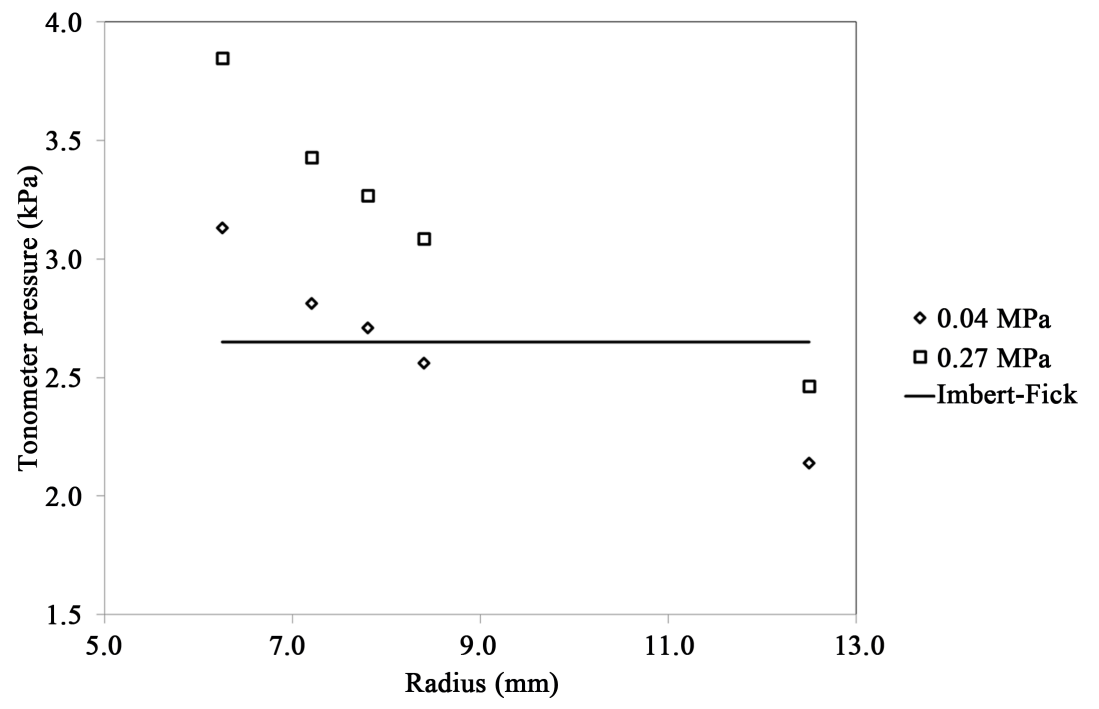

Figure 17. Effect of radius of curvature, using cornea shell properties of Equation (12) at an IOP of $2.6 \mathrm{kPa}$. The key defines values of $G_{r \theta}$.

\section{Conclusions}

A mathematical model describing the working mechanism of Goldmann-type applanation tonometers has been developed. The models consist of a hollow elastic shell of orthotropic elastic material, representing the sclera and cornea, containing an elastic liquid core representing the vitreous body. The models, once pressurised, are compressed across a diameter against a rigid flat plane, with the contact area increasing as the compression continues. The finite element approach allows the IOP to be compared with the predictions of it deduced via tonometry and the Imbert-Fick law-the tonometer pressures.

The mechanical behaviours of the sclera and cornea are crucial to the analysis. Given the stiffness values taken from tensile data in the literature, it becomes clear that the assumption of isotropy is entirely inappropriate. The shell of the model eye is assumed to be orthotropic, and as far as possible its elastic constants are taken from published measurements. 
The tonometer pressures are approximately a linear function of IOP with a positive intercept (Figure 12 and Figure 16). The value of the intercept can be interpreted as the tonometer pressure at zero IOP, i.e. the pressure required to flatten the outer elastic shell. The slope is much less than unity, the value that would apply if the Imbert-Fick law were valid. The Imbert-Fick law may be approximately valid at some pressures. However, since the tonometer pressures depend on the elastic properties of the outer shell and hence the cornea, the values of which are not fully defined, this cannot be established with certainty.

Results with the present modelling approach could be improved by the acquisition of more precise cornea and sclera elastic properties. The method can be trivially extended to more complex geometries, exploring variables such as corneal thickness and incorporating non-axisymmetric effects. Also, pressure rather than displacement boundary conditions could be applied to model alternative tonometry methods. Finally, the effects of scleral buckling could also be investigated using models of this kind.

\section{References}

[1] Fatt, I. and Weissman, B.A. (1992) Physiology of the Eye: An Introduction to Vegetative Functions. 2nd Edition, Butterworth-Heinemann, London.

[2] Bron, A.J., Tripathy, R.C. and Tripathy, B.J. (1997) Wolff's Anatomy of the Eye and Orbit. Chapman and Hall Medical, London.

[3] Jayasuriya, A.C., Ghosh, S., Scheinbeim, J.I., Lubkin, V., Bennett, G. and Kramer, P.A. (2003) A Study of Piezoelectric and Mechanical Anisotropies of the Human Cornea. Biosensors and Bioelectronics, 18, 381-387. http://dx.doi.org/10.1016/S0956-5663(02)00144-6

[4] Imbert, A. (1885) Théorie sur ophthaltonomètres. Archives of Ophthalmology, 5, 358-363.

[5] Fick, A. (1888) Ueber messung des druckes in auge. Archiv für die Gesamte Physiologie des Menschen und der Tiere, 42, 86-90. http://dx.doi.org/10.1007/BF01669349

[6] Elsheikh, A. and Wang, D. (2007) Numerical Modelling of Corneal Biomechanical Behaviour. Computer Methods in Biomechanics and Biomedical Engineering, 10, 85-95. http://dx.doi.org/10.1080/10255840600976013

[7] Goldmann, H. and Schmidt, T. (1957) Uber applanations-tonometrie. Ophthalmologia, 134, 221-242. http://dx.doi.org/10.1159/000303213

[8] Ehlers, N., Bramsen, T. and Sperling, S. (1975) Applanation Tonometry and Central Corneal Thickness. Acta Ophthalmologica (Copenh), 53, 34-43. http://dx.doi.org/10.1111/j.1755-3768.1975.tb01135.x

[9] Whitacre, M.M., Stein, R.A. and Hassanein, K. (1993) The Effect of Corneal Thickness on Applanation Tonometry. American Journal of Ophthalmology, 115, 592-596. http://dx.doi.org/10.1016/S0002-9394(14)71455-2

[10] Benham, P.P., Crawford, R.J. and Armstrong, C.G. (1996) Mechanics of Engineering Materials. 2nd Edition, Prentice Hall, Pearson Education Ltd., Essex.

[11] Orssengo, G.J. and Pye, D.C. (1999) Determination of the True Intraocular Pressure and Modulus of Elasticity of the Human Cornea in Vivo. Bulletin of Mathematical Biology, 61,551-572. http://dx.doi.org/10.1006/bulm.1999.0102

[12] Śródka, W. (2010) Goldmann Applanation Tonometry-Not as Good as Gold. Acta of Bioengineering and Biomechanics, 12, 39-47.

[13] Buzard, K.A. (1992) Introduction to Biomechanics of the Cornea. Refractive \& Corneal Surgery, 8, 127-138.

[14] Asejczyk-Widlicka, M., Śródka, W., Schachar, R.A. and Pierścionek, B.K. (2011) Material Properties of the Cornea and Sclera: A Modelling Approach to Test Experimental Analysis. Journal of Biomechanics, 44, 543-546.

http://dx.doi.org/10.1016/j.jbiomech.2010.09.032

[15] Elsheikh, A., Wang, D., Kotecha, A., Brown, M. and Garway-Heath, D. (2006) Evaluation of Goldmann Applanation Tonometry Using a Nonlinear Finite Element Ocular Model. Annals of Biomedical Engineering, 34, 1628-1640. http://dx.doi.org/10.1007/s10439-006-9191-8

[16] Singh, D., Firouzbakhsh, K. and Ahmadian, T. (2011) Effect of the Human Eye Cornea Material Property on Measurement of the Intraocular Eye Pressure Utilizing Goldmann Applanation Tonometer. AMAE International Journal on Manufacturing and Material Science, 1, 1-4.

[17] Pandolfi, A. and Manganiello, F. (2006) A Model for the Human Cornea: Constitutive Formulation and Numerical Analysis. Biomechanics and Modeling in Mechanobiology, 5,237-246. http://dx.doi.org/10.1007/s10237-005-0014-x

[18] Girard, M.J.A., Downs, J.C., Bottlang, M., Burgoyne, C.F. and Suh, J.-K.F. (2009) Peripapillary and Posterior Scleral Mechanics, Part II-Experimental and Inverse Finite Element Characterization. ASME Journal of Biomechanical Engineering, 131, Article ID: 051012. http://dx.doi.org/10.1115/1.3113683 
[19] Battaglioli, J.L. and Kamm, R.D. (1984) Measurements of the Compressive Properties of Scleral Tissue. Investigative Ophthalmology \& Visual Science, 25, 59-65.

[20] ABAQUS 6.12 (2013) Dassault Systemes Simulia Corp., Rising Sun Mills, Providence.

[21] Friberg, T.R. and Lace, J.W. (1988) A Comparison of the Elastic Properties of Human Choroid and Sclera. Experimental Eye Research, 47, 429-436. http://dx.doi.org/10.1016/0014-4835(88)90053-X

[22] Keeling, S.L., Propst, G., Stadler, G. and Wackernagel, W. (2009) A Mathematical Model for the Deformation of the Eyeball by an Elastic Band. Mathematical Medicine and Biology, 26, 165-185. http://dx.doi.org/10.1093/imammb/dqp008

[23] Schulz, D.S., Jeffrey, C.L., Lee, S.M., Trinidad, M.L. and Stewart, J.M. (2008) Structural Factors That Mediate Scleral Stiffness. Investigative Ophthalmology \& Visual Science, 49, 4232-4236.

[24] Zeng, Y., Yang, J., Huang, K., Lee, Z. and Lee, X. (2001) A Comparison of Biomechanical Properties between Human and Porcine Cornea. Journal of Biomechanics, 34, 533-537. http://dx.doi.org/10.1016/S0021-9290(00)00219-0

[25] Hoeltzel, D.A., Altman, P., Buzard, K. and Choe, K. (1992) Strip Extensiometry for Comparison of the Mechanical Response of Bovine, Rabbit and Human Corneas. Journal of Biomechanical Engineering, 114, 202-215. http://dx.doi.org/10.1115/1.2891373

[26] Ward, I.M. and Sweeney, J. (2013) Mechanical Properties of Solid Polymers. 3rd Edition, Wiley, Chichester, 168-170.

[27] Li, L.-Y. and Tighe, B.J. (2006) The Anisotropic Material Constitutive Models for the Human Cornea. Journal of Structural Biology, 153, 223-230. http://dx.doi.org/10.1016/j.jsb.2005.10.014 